\title{
CrkL is required for donor T cell migration to GvHD target organs
}

\author{
Nathan H. Roy ${ }^{1}$, Mahinbanu Mammadli ${ }^{2}$, Janis K. Burkhardt ${ }^{1}$ and Mobin Karimi ${ }^{2}$ \\ ${ }^{1}$ Department of Pathology and Laboratory Medicine, Children's Hospital of Philadelphia Research Institute and Perelman \\ School of Medicine at the University of Pennsylvania, Philadelphia, PA 19104, USA \\ ${ }^{2}$ Department of Microbiology and Immunology, SUNY Upstate Medical University, Syracuse, NY 13210, USA \\ Correspondence to: Mobin Karimi, email: karimim@upstate.edu \\ Keywords: graft-versus-host disease; CrkL; T cell; migration; inflammation \\ Received: January 03, $2020 \quad$ Accepted: February 17, $2020 \quad$ Published: April 28, 2020 \\ Copyright: Roy et al. This is an open-access article distributed under the terms of the Creative Commons Attribution License 3.0 \\ (CC BY 3.0), which permits unrestricted use, distribution, and reproduction in any medium, provided the original author and source \\ are credited.
}

\section{ABSTRACT}

The success of cancer therapies based on allogeneic hematopoietic stem cell transplant relies on the ability to separate graft-versus-host disease (GvHD) from graft-versus-tumor (GVT) responses. Controlling donor $\mathrm{T}$ cell migration into peripheral tissues is a viable option to limit unwanted tissue damage, but a lack of specific targets limits progress on this front. Here, we show that the adaptor protein CrkL, but not the closely related family members CrkI or CrkII, is a crucial regulator of $T$ cell migration. In vitro, CrkL-deficient $\mathrm{T}$ cells fail to polymerize actin in response to the integrin ligand ICAM-1, resulting in defective migration. Using a mouse model of GvHD/GVT, we found that while CrkL-deficient $T$ cells can efficiently eliminate hematopoietic tumors they are unable to migrate into inflamed organs, such as the liver and small intestine, and thus do not cause GvHD. These results suggest a specific role for CrkL in trafficking to peripheral organs but not the lymphatic system. In line with this, we found that although CrkL-deficient $\mathrm{T}$ cells could clear hematopoietic tumors, they failed to clear the same tumor growing subcutaneously, highlighting the role of CrkL in controlling $\mathrm{T}$ cell migration into peripheral tissues. Our results define a unique role for CrkL in controlling $\mathrm{T}$ cell migration, and suggest that CrkL function could be therapeutically targeted to enhance the efficacy of immunotherapies involving allogeneic donor cells.

\section{INTRODUCTION}

$\mathrm{T}$ cell migration out of the vasculature into peripheral tissue is a key control point in the inflammatory response. Although trafficking to tissues is required for protective immunity, uncontrolled $\mathrm{T}$ cell infiltration into tissue can result in exacerbated inflammation and tissue damage. $\mathrm{T}$ cell recruitment is highly regulated by the local endothelial cells which, in response to pro-inflammatory cues, up-regulate molecules such as chemokines and adhesion receptors. These molecules promote $\mathrm{T}$ cell adhesion to the endothelial monolayer, and guide migration through the monolayer into the tissue [1]. The endothelial ligands ICAM-1 and VCAM-1 are recognized by the $\mathrm{T}$ cell integrins LFA-1 and VLA4 , respectively, which mediate firm adhesion to the vascular wall and subsequent migration into inflamed tissue $[1,2]$. In addition to acting as adhesion receptors, integrins also initiate downstream signaling events that are important for $\mathrm{T}$ cell migration. For instance, ligation of LFA-1 immediately results in T cell polarization, actin polymerization, and migration, all of which are necessary for crossing the endothelial barrier [3-7]. Integrin blocking antibodies have been used successfully to treat multiple sclerosis and other inflammatory diseases, but this approach interferes with a broad range of responses. Disruption of signaling events downstream of engaged integrins could yield more context-specific therapies, but to develop such strategies, a better understanding of the relevant signaling pathways is required.

We recently identified the CT10 Regulator of Kinase (Crk) family of adapter proteins as crucial intermediaries that link LFA-1 engagement to actin responses and migration in T cells [7]. Activated T cells lacking Crk 
proteins fail to polymerize actin downstream of LFA-1, and migrate slower and less directionally than their WT counterparts [7]. Importantly, these mutant $\mathrm{T}$ cells also have defects in crossing endothelial monolayers in vitro, and they fail to traffic to sites of inflammation in vivo [8]. Additionally, using a clinically relevant mouse model of graft-versus-host disease/graft-versus-leukemia (GvHD/ GVL), we showed that $\mathrm{T}$ cells lacking Crk proteins can efficiently clear tumor cells but cause little-to-no GvHD pathology [8]. Consistent with the view that Crk proteins mediate integrin-dependent trafficking in a tissue-specific manner, we found that Crk deficient $\mathrm{T}$ cells could not migrate to the target GvHD organs liver and small intestine (SI), although they trafficked efficiently to the secondary lymphoid organs spleen and lymph nodes. These findings identify Crk proteins as potential targets for tissue-selective disruption of integrin-dependent inflammatory responses.

Development of Crk proteins as therapeutic targets requires addressing redundancy among family members. The Crk family consists of three isoforms transcribed from two loci. CrkI and CrkII are transcribed from the $c r k$ locus, while the paralog CrkL is transcribed from the $c r k l$ locus. These proteins are composed of a single N-terminal $\mathrm{SH} 2$ domain, followed by either two consecutive $\mathrm{SH} 3$ domains (CrkII and CrkL), or a single SH3 domain (CrkI) [9]. The Crk proteins are widely expressed across tissues and have many biological functions, all of which stem from their role as adaptor proteins that coordinate signaling complexes downstream of cell surface receptors $[9,10]$. Crk proteins are particularly important for adhesion and migration [11]. They have been shown to localize to adhesion sites and regulate the stability of these structures in non-hematopoietic cells [12-15], and alterations in their expression is associated with invasive potential in several tumors [16-18]. Crk family members share many of the same binding partners, and they have been shown to have overlapping functions in some processes $[15,19,20]$. On the other hand, there are clear instances (most notably during development) where the Crk proteins have non-overlapping roles, showing that in some instances they have evolved separate functions $[21,22]$. All of our previous work defining the role of Crk proteins in $\mathrm{T}$ cell migration was performed using a mouse floxed for both the crk and crkl loci and crossed with a CD4-cre mouse, resulting in T cells devoid for all three family members (herein referred to as DKO). Therefore, it is unclear if the Crk family members work together to promote $\mathrm{T}$ cell migration, or if only a single Crk isoform is responsible for this function. Now, using T cells lacking either CrkI/II or CrkL, we show that CrkL is the dominant Crk family member that controls $\mathrm{T}$ cell migration. T cells lacking CrkI/II show a WT phenotype, whereas T cells lacking CrkL phenocopy DKO T cells in their responses to ICAM-1 in vitro and in vivo in a GvHD/GVT mouse model. This work has defined a unique role for CrkL in
$\mathrm{T}$ cell migration, opening the door to novel therapeutic approaches based on targeting CrkL function.

\section{RESULTS}

\section{CrkL is needed for $T$ cell spreading and migration in response to the integrin ligand ICAM-1}

We showed previously that $\mathrm{T}$ cells lacking all Crk family members exhibit defects in integrin-dependent migration and in vivo trafficking $[7,8]$. To ask if this aspect of Crk protein function depends on a single protein isoform or if there is functional redundancy among family members, we used mice that are floxed for either the $c r k$ or the $c r k L$ locus, crossed with CD4-Cre mice to specifically delete these loci in $\mathrm{T}$ cells. The resulting $\mathrm{T}$ cells are lacking either CrkI/II or CrkL (Figure 1A). We first tested the ability of these $T$ cells to polymerize actin and migrate in response to surface-presented integrin ligands in vitro. Rested $\mathrm{CD}^{+} \mathrm{T}$ cell blasts were prepared as described in Materials and Methods, and then allowed to interact with ICAM-1 coated surfaces, fixed, and labelled with phalloidin to visualize F-actin. As shown previously [4, 5, 7], WT T cells adhered tightly to the surfaces, and formed a polarized morphology characteristic of cells migrating on ICAM-1 (Figure 1B). These WT cells showed strong actin polymerization, especially at the leading edge (Figure 1B). T cells lacking CrkI/II were indistinguishable from the WT cells. In contrast, DKO and CrkL KO T cells failed to spread, and lacked a clear F-actin-rich leading edge (Figure 1B). Quantitation of the total F-actin signal confirmed that DKO and CrkL KO T cells responding to ICAM-1 contained significantly less F-actin than WT or CrkI/II KO T cells (Figure 1C). To determine if the defects in actin responses result in altered migration, we imaged $\mathrm{T}$ cells migrating on ICAM-1 coated surfaces and tracked cell movement. DKO and CrkL KO T cells migrated significantly slower than WT or CrkI/II KO cells (Figure 1D). As we showed previously for DKO T cells [7], loss of a clear actin-rich leading edge also resulted in reduced directional persistence in CrkL KO $\mathrm{T}$ cells (data not shown). Together, these data show that the two products of the Crk locus, CrkI and CrkII, are dispensable for actin polymerization and migration downstream of integrin engagement. The CrkL protein is the relevant family member controlling these events in T cells.

\section{T cells lacking CrkL clear hematopoietic tumors but fail to traffic to target GvHD organs}

To ask if CrkL is also the critical Crk family member responsible for $\mathrm{T}$ cell migration in vivo, we utilized a graft-versus-host/graft-versus-tumor mouse model (GvHD/GVT). In this model, GvHD occurs in a subset of organs and involves early migration of 
alloreactive $\mathrm{T}$ cells into these organs followed by $\mathrm{T}$ cell expansion and later tissue destruction [23]. To test the role of the individual Crk proteins in GvHD pathology, lethally irradiated $\mathrm{BALB} / \mathrm{c}$ mice were injected with T-cell depleted C57BL/6 bone marrow (TCDBM) along with $\mathrm{CD}^{+} \mathrm{T}$ cells from WT, DKO, CrkI/II KO, or CrkL KO C57BL/6 mice. In addition, A20 lymphoma cells expressing luciferase were also injected, to determine if the transferred $\mathrm{CD}^{+} \mathrm{T}$ cells could eliminate tumor cells. Mouse weights, GvHD clinical score, tumor burden, and overall survival were monitored. As expected, transfer of WT CD ${ }^{+} \mathrm{T}$ cells caused severe GvHD with no detectable signs of tumors at the time of sacrifice (Figure 2A-2D). As we showed previously [8], $\mathrm{DKO} \mathrm{CD}^{+} \mathrm{T}$ cells did not cause GvHD, but still efficiently cleared the tumor cells, resulting in healthy mice throughout the course of the experiment. CrkL $\mathrm{KO} \mathrm{CD} 8^{+} \mathrm{T}$ cells recapitulated the DKO phenotype, while CrkI/II $\mathrm{KO} \mathrm{CD}^{+} \mathrm{T}$ cells had a phenotype indistinguishable from the WT. This pattern was observed for all disease metrics, including overall survival (Figure 2A-2D).

Based on the failure of CrkL KO T cells to migrate normally in vitro, we reasoned that CrkL KO T cells do not cause GvHD because of their inability to traffic to target GvHD organs. However, because the GvHD/GVT experiments take place over the course of several weeks, it is difficult to establish a causative role for T cell migration in initiating the inflammatory response. We therefore set up a short-term competitive experiment to examine the role of Crk proteins in T cell migration into target GvHD organs. Lethally irradiated BALB/c mice were injected with TCDBM along with a $1: 1$ mix of WT H-2K $\mathrm{K}^{\mathrm{b}+}$ CD $45.1^{+}$ $\mathrm{CD}^{+} \mathrm{T}$ cells (purified from B6. SJL mice) and either WT, $\mathrm{DKO}, \mathrm{CrkI} / \mathrm{II} \mathrm{KO}$, or CrkL KO CD8 ${ }^{+} \mathrm{T}$ cells, all of which are $\mathrm{H}-2 \mathrm{~K}^{\mathrm{b}+} \mathrm{CD} 45.2^{+}$. Seven days post injection, before the onset of GvHD pathology, the mice were sacrificed and their livers, small intestines, and spleens were processed for flow cytometric analysis. A representative gating strategy to identify donor $\mathrm{H}-2 \mathrm{~K}^{\mathrm{b}+} \mathrm{CD} 8^{+} \mathrm{T}$ cells is shown in Figure $3 \mathrm{~A}$. As expected, when WT CD $45.1^{+} \mathrm{T}$ cells were mixed with control WT CD45.2 $2^{+} \mathrm{T}$ cells, the two were detected in approximately a 1:1 ratio in every organ tested (Figure 3B and 3C). This was also true for CrkI/II KO T cells, indicating that Crk I and Crk II are dispensable for $\mathrm{T}$ cell migration in vivo. However, when $\mathrm{DKO}$ or $\mathrm{CrkL}$ KO T cells were used, these $\mathrm{T}$ cells had a significant defect in migration to the liver and the small intestine compared to their CD45.1 $1^{+}$WT counterparts. Importantly, migration into the spleen was largely normal, indicating that DKO and CrkL KO T cells can still traffic efficiently to lymphoid organs (Figure 3B and 3C). Taken together with the data in Figures 1 and 2, these results demonstrate that CrkL is the relevant Crk family protein controlling $\mathrm{T}$ cell migration both in vitro and in vivo, and highlights the importance of CrkL-dependent $\mathrm{T}$ cell trafficking during inflammation and GvHD.

\section{CrkL KO T cells fail to eliminate a subcutaneous tumor}

The trafficking studies above suggest that the ability of CrkL KO T cells to clear lymphoma cells while inducing minimal GvHD pathology stems from their ability to migrate efficiently to lymphoid tissues where the tumor resides, but not to GvHD target organs. If so, one would predict that if the tumor were located in a peripheral tissue outside of the lymphatic system, CrkL KO T cells would not be able to access and kill the tumor. To test this, we set up a GvHD/GVT experiment similar to that in Figure 2, except that the A20 tumor cells were injected subcutaneously as opposed to intravenously. As shown in Figure 4, both WT and CrkI/II KO T cells
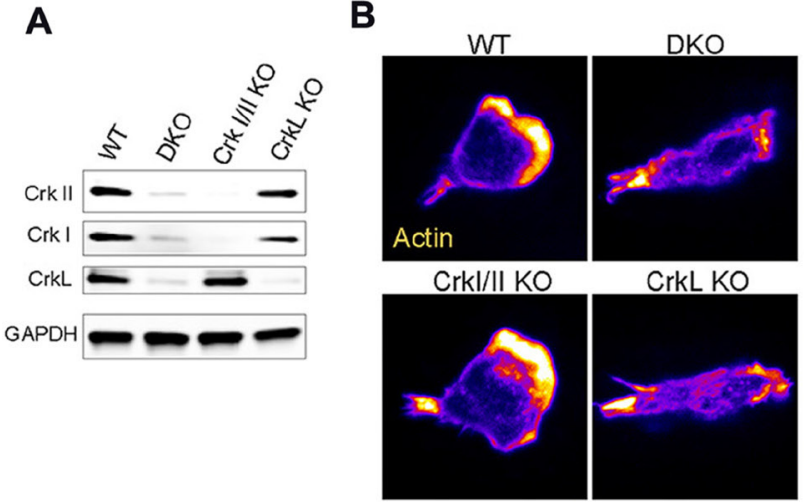
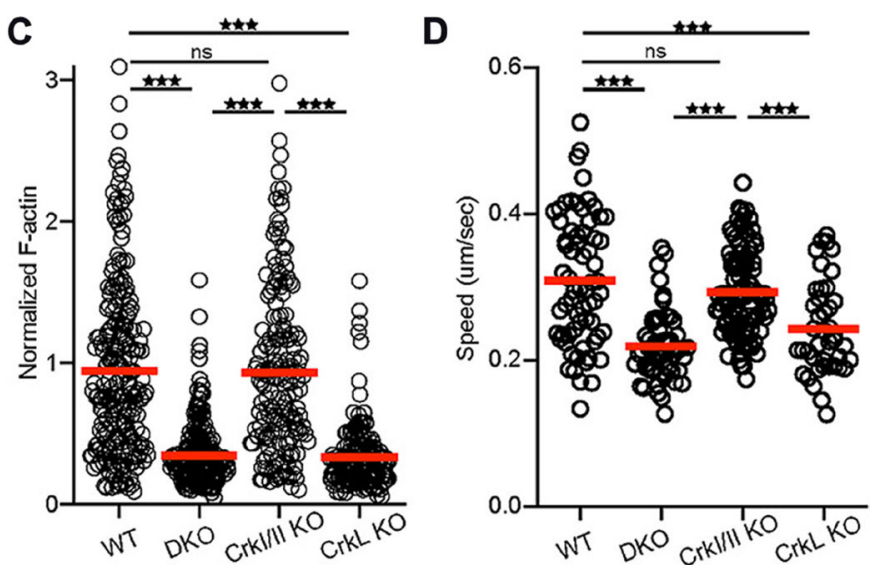

Figure 1: CrkL is required for $\mathbf{T}$ cell integrin responses. (A) Western blot of $\mathrm{T}$ cells purified from WT or knockout mice. Images were taken using a Licor Odyssey western blot imaging system and image was cropped to display the indicated proteins. (B) Activated T cells from the indicated genotypes were allowed to settle on ICAM-1 coated coverslips, fixed, and stained for F-actin using fluorescent phalloidin. (C) Quantification of total F-actin signal from (B), pooled from $n=3$. (D) Activated T cells from the indicated genotypes were imaged migrating on ICAM-1 coated surfaces and average speed was calculated, pooled from 3 independent experiments. Cells were purified from one mouse per genotype per experiment. A one-way ANOVA was used to calculate $p$-values. ${ }^{*} p<0.05 ;{ }^{* *} p<0.01 ;{ }^{* * *} p<0.001$. 
efficiently cleared the subcutaneous tumor, but these animals succumbed to GvHD. In contrast, DKO and CrkL KO T cells were unable to clear the subcutaneous tumor and these recipients were sacrificed due to the resulting tumor pathology (Figure 4A-4D). Together, these data indicate that deletion of CrkL in $\mathrm{T}$ cells can separate GvHD from GVT effects, but only for tumors that reside in the circulation and in secondary lymphoid organs (e. g. hematologic malignancies). More broadly, these data provide strong evidence that the CrkL controls $\mathrm{T}$ cell entry into inflamed tissues, and that disruption of CrkL function can be used to perturb this trafficking, while preserving trafficking to lymphoid organs.

\section{DISCUSSION}

We showed previously that $\mathrm{T}$ cells lacking all Crk family proteins exhibit defective migration into inflamed tissue in vivo, and an inability to carry out transendothelial migration (TEM) in vitro [8]. Interestingly, this in vitro phenotype could be rescued by overexpression of any Crk family member [8], raising the possibility that Crk proteins might exhibit functional redundancy with respect to $\mathrm{T}$ cell migration. Here, we have carried out more stringent in vitro and in vivo analysis using single and double knockout $\mathrm{T}$ cells. We show that CrkL, but not CrkI or CrkII, is required for $\mathrm{T}$ cell actin polymerization and migration in response to ICAM-1. In an in vivo GvHD/ GVT model, CrkL deficient $\mathrm{T}$ cells failed to traffic to GvHD target organs and did not cause inflammation, but could efficiently clear hematopoietic tumor cells. However, CrkL KO T cells could not clear the same tumor when it was growing subcutaneously, highlighting the role of CrkL in controlling $\mathrm{T}$ cell migration into peripheral tissues. Taken together, our studies identify a unique role for CrkL in $\mathrm{T}$ cell migration in vitro and in vivo, opening
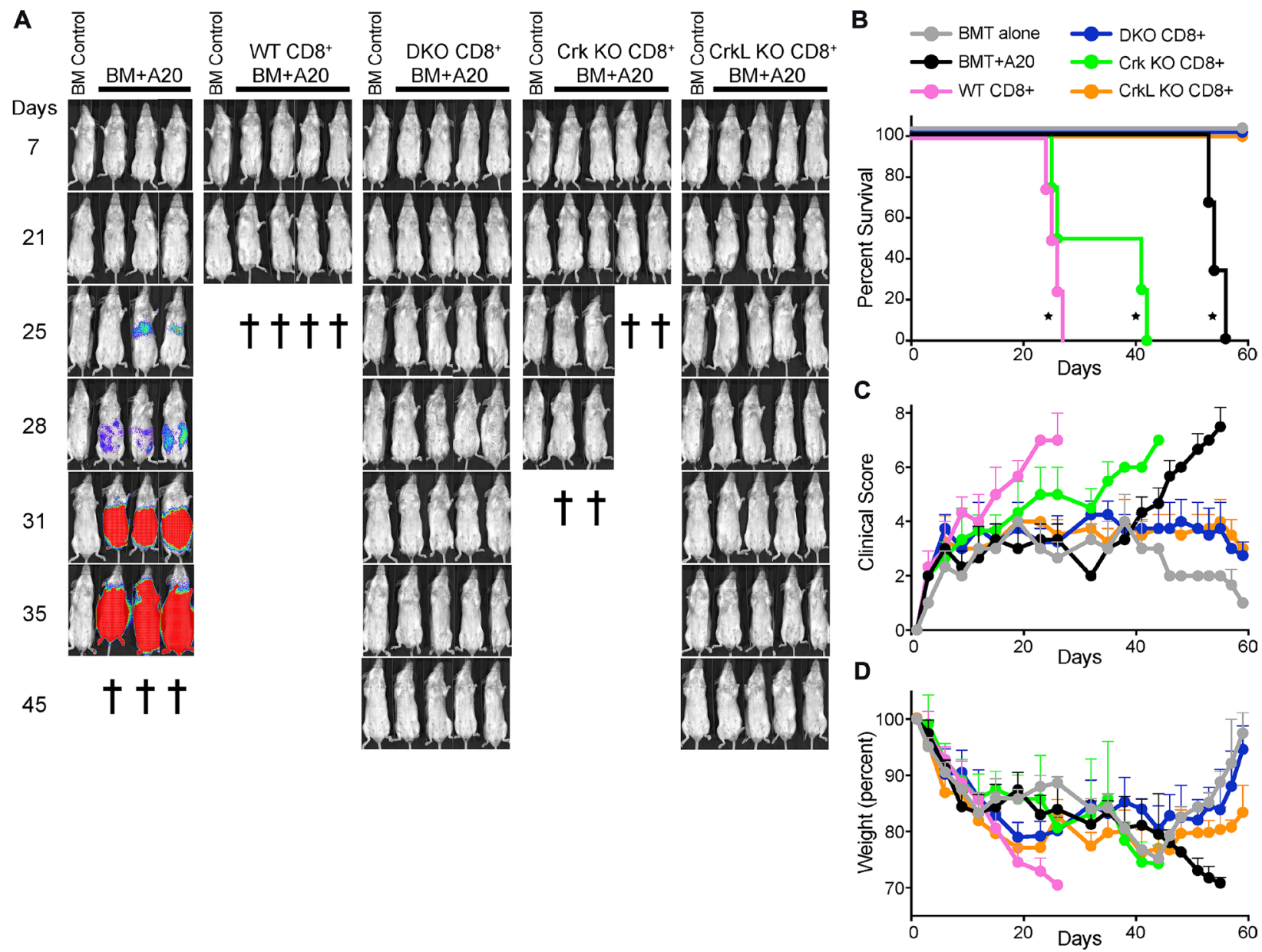

Figure 2: T cells lacking CrkL do not cause pathology in a model of GvHD/GVL. Lethally irradiated BALB/c mice served as recipients in this transplant model. As a control, irradiated mice were injected intravenously with T-cell depleted C57BL/6 bone marrow alone (BM control). For experimental groups, irradiated mice were injected intravenously with $\mathrm{T}$ cell depleted C57BL/6 bone marrow along with A20 lymphoma cells expressing luciferase with no added T cells (BM+A20), or with CD8 ${ }^{+} \mathrm{T}$ cells from WT, DKO, CrkI/II $\mathrm{KO}$, or CrkL KO mice. (A) Over the next several weeks, the mice were periodically injected with luciferin and imaged to visualize tumor burden. The cross symbols represent when each individual mouse was sacrificed due to either high tumor burden or severe GvHD. (B-D) Quantification of clinical data from the mice shown in panel A. (B) Overall survival ( ${ }^{*} p<0.05$, Wilcoxon test), (C) clinical score, and (D) mouse weights. One representative experiment of $n=3$ experiments, with 7 mice per group per experiment. 
the door for future studies aimed at disrupting CrkL biology to alter $\mathrm{T}$ cell function.

Crk proteins have long been associated with adhesion and migration in many cell types including fibroblasts, hematopoietic cell lines, and cancer cells $[10,11,18]$. They fulfill this function by linking tyrosine phosphorylated proteins to effector proteins that govern adhesion and migration, such as Src and Abl family kinases, GEFs and GAPs $[9,10]$. In this way, Crk proteins coordinate multi-protein complexes downstream of cell surface receptors. In $\mathrm{T}$ cells, only a small number of proteins are known to interact with Crk proteins [24].

A

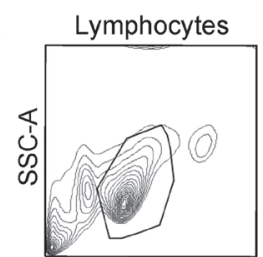

FSC-A

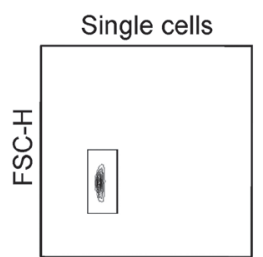

FSC-W

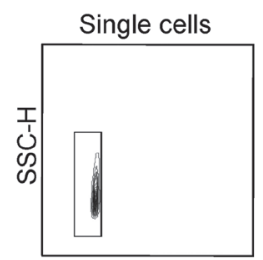

SSC-W

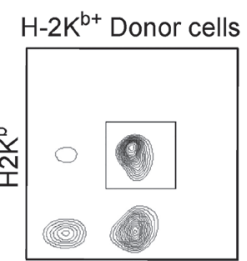

CD3

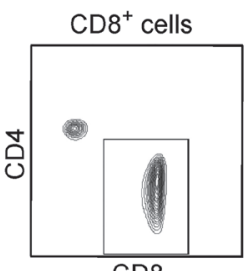

CD8

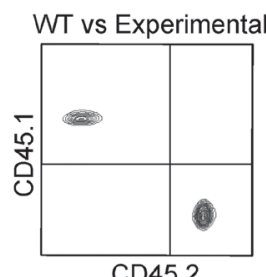

CD45.2

B

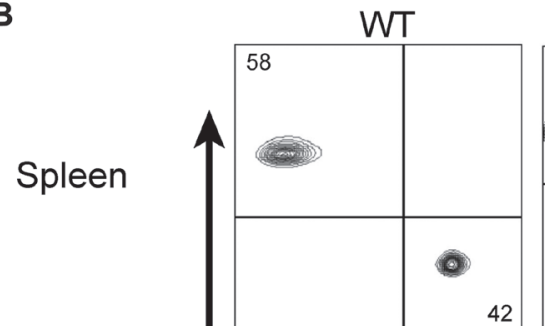

DKO
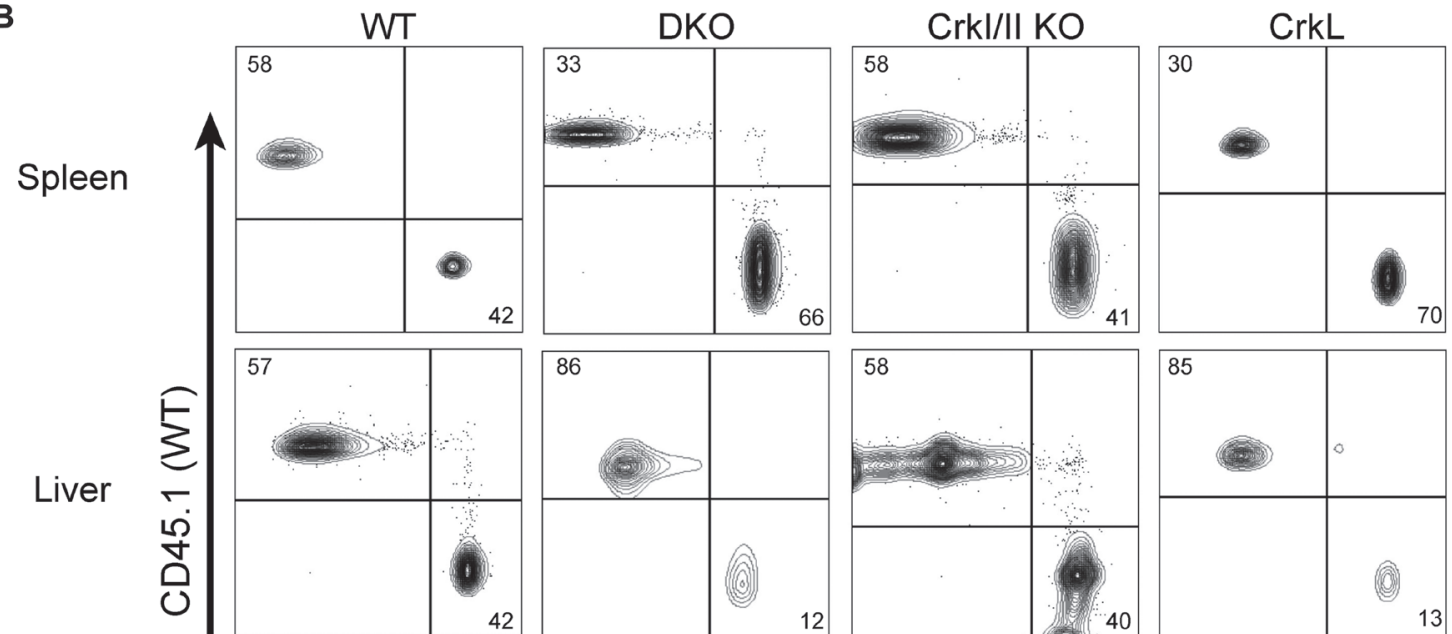

42
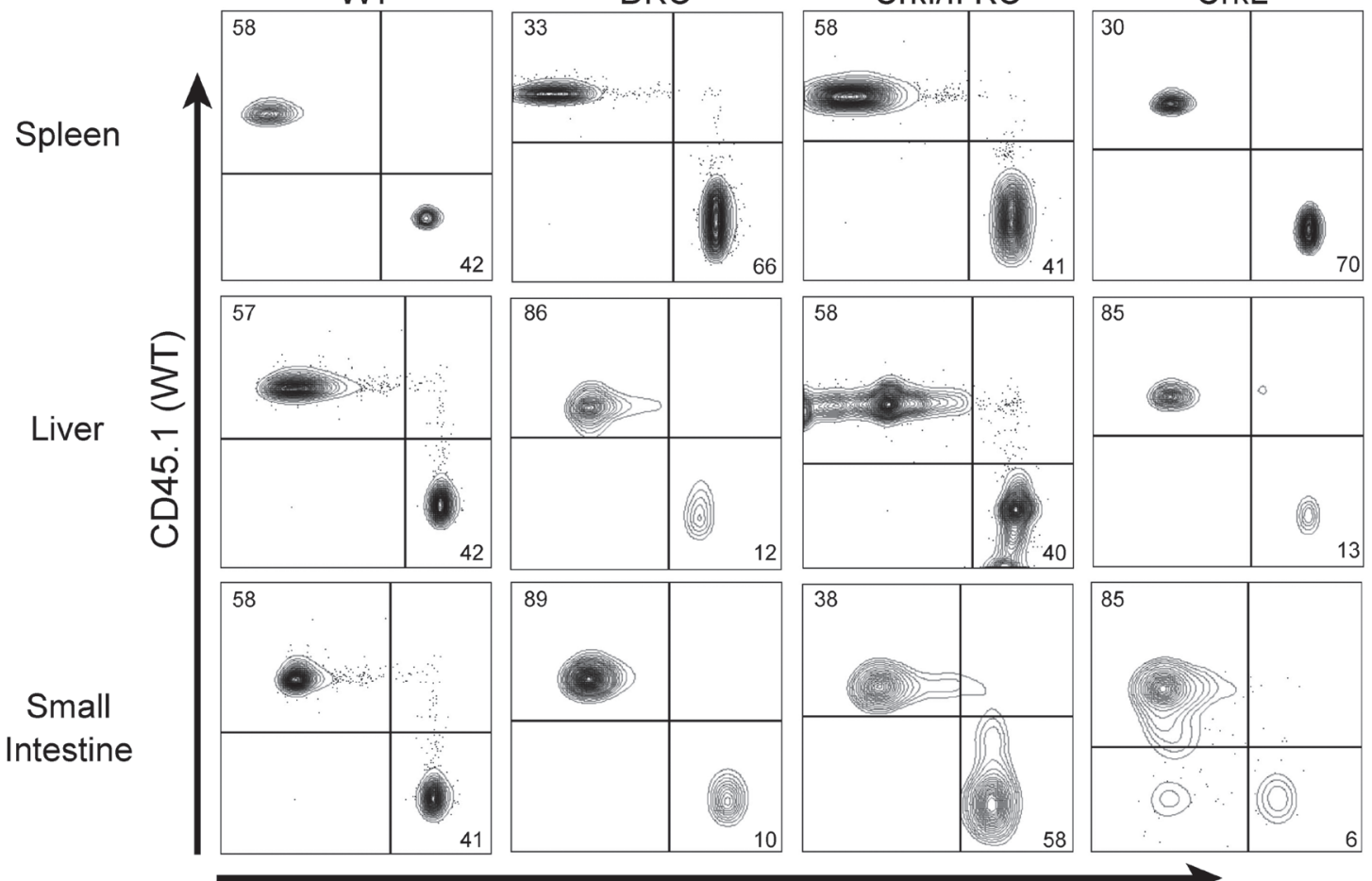

CD45.2

C Spleen
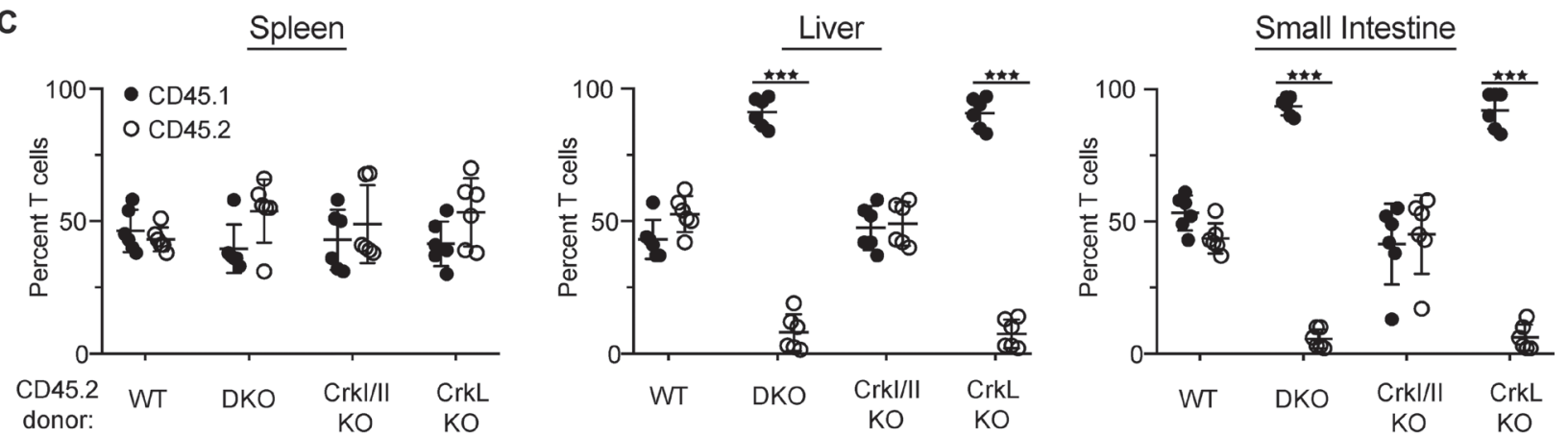

Figure 3: T cell trafficking to GvHD organs required CrkL. Lethally irradiated BALB/c (H-2K $\left.\mathrm{K}^{\mathrm{d}}\right)$ mice were injected with T-cell depleted C57BL/6 bone marrow along with a 1:1 ratio of $\mathrm{H}-2 \mathrm{~K}^{\mathrm{b}+} \mathrm{WT} \mathrm{CD} 45.1^{+} \mathrm{CD}^{+} \mathrm{T}$ cells and $\mathrm{H}-2 \mathrm{~K}^{\mathrm{b}+} \mathrm{CD} 45.2^{+} \mathrm{CD} 8^{+} \mathrm{T}$ cells from either WT, DKO, CrkI/II KO, or CrkL KO mice. Seven days post injection, the mice were sacrificed and the spleen, liver, and small intestines were processed for flow cytometry. (A) Representative gating strategy to identify donor CD8 ${ }^{+} \mathrm{T}$ cells. (B) A single mouse from a representative experiment is shown. (C) Quantification of CD45.1 $1^{+}$to CD45.2 $2^{+}$percentages from indicated tissues. Two-way ANOVA, ${ }^{* * *} p<0.001 . n=3$ experiments, with 7 mice per group per experiment. 
Based on our data defining a clear role for CrkL in actin polymerization and $\mathrm{T}$ cell migration, it is likely that CrkL has unique binding partners in $\mathrm{T}$ cells that mediate these phenotypes. The isolated $\mathrm{SH} 2$ and $\mathrm{SH} 3$ domains of CrkII and CrkL are very similar and have nearly identical binding affinities for protein ligands [25]. Nonetheless, the full-length proteins show significant differences. In the intact protein, the $\mathrm{SH} 2$ domain of CrkII binds efficiently to tyrosine phosphorylated ligands, whereas the $\mathrm{SH} 2$ domain of CrkL shows low binding activity. Conversely, the N-terminal SH3 domain of CrkL binds freely to proline-rich peptides, but this activity is largely masked in CrkII [25]. Further differences are seen upon tyrosine phosphorylation of Crk proteins, where the phosphorylation of CrkII (but not CrkL) further reduces binding to proline-rich ligands. Ligand binding of CrkII is also regulated by proline isomerization [26-28]. Since the relevant proline is absent from CrkL, this likely represents another structural difference that regulates isoformspecific binding. Future work aimed at determining the specific binding partners of the different Crk proteins in primary $\mathrm{T}$ cells under different phosphorylation and isomerization conditions will be crucial for formulating therapies that alter CrkL dependent signaling pathways to tune $\mathrm{T}$ cell function.

We show that CrkL KO T cells fail to enter inflamed tissues and subcutaneous tumors, but they efficiently access lymphoid tissues. The selective requirement for CrkL in entering inflamed tissue likely reflects the requisite role of $\mathrm{CrkL}$ in regulating actin dynamics downstream of integrin engagement. $\mathrm{T}$ cells actively migrate on inflamed postcapillary venules and make numerous actin-dependent invadopodia-like protrusions to push their way through the endothelial monolayer
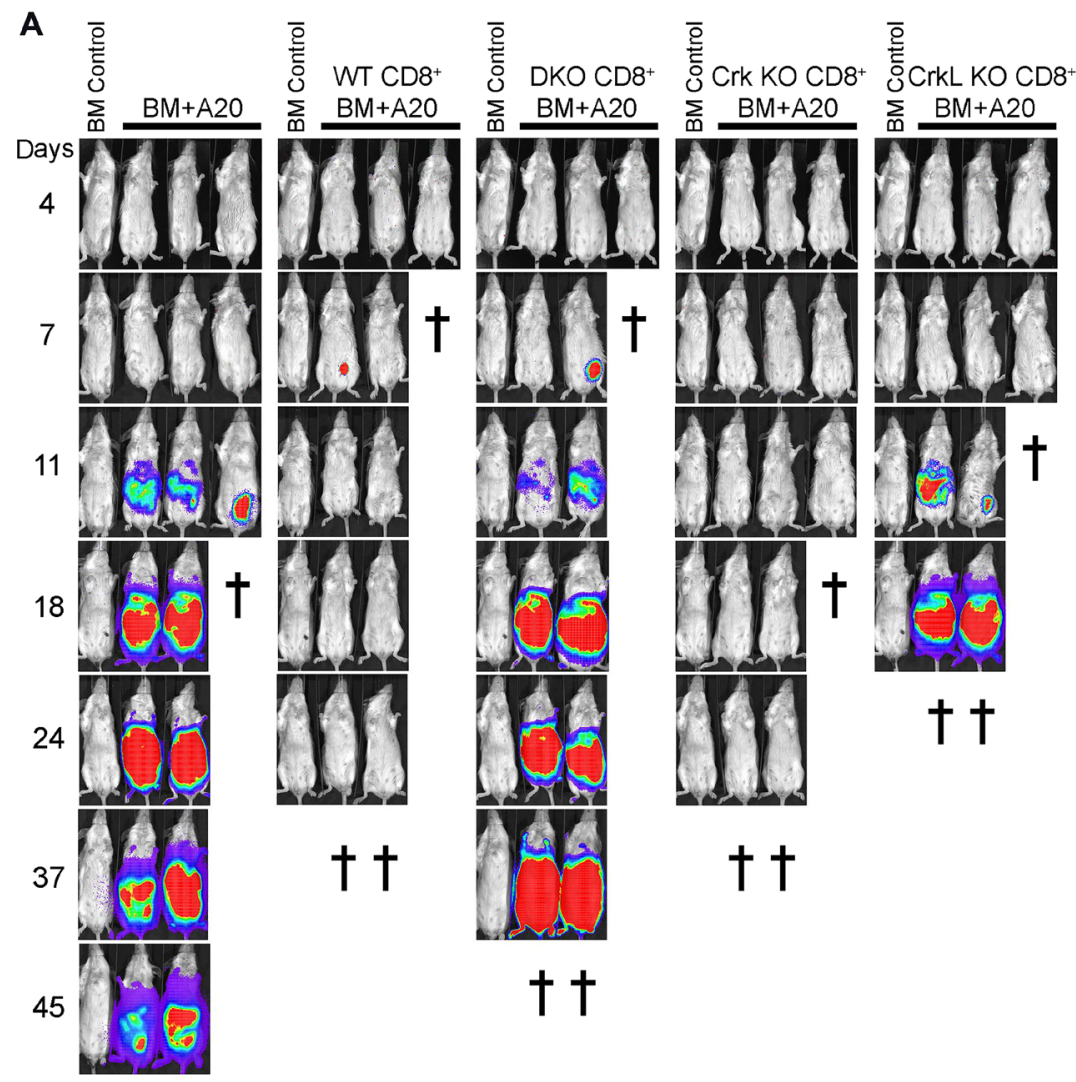

B
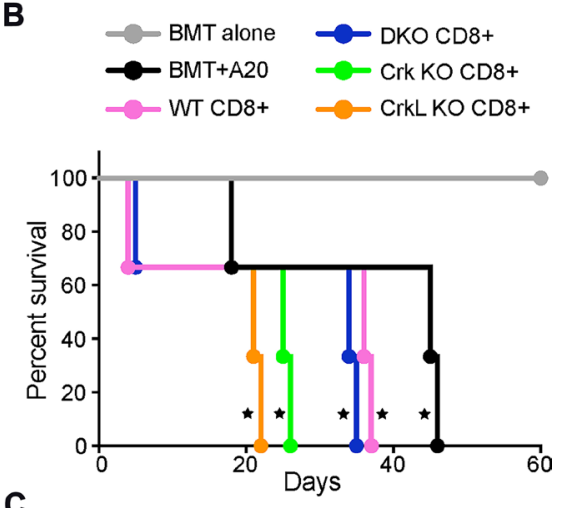

C

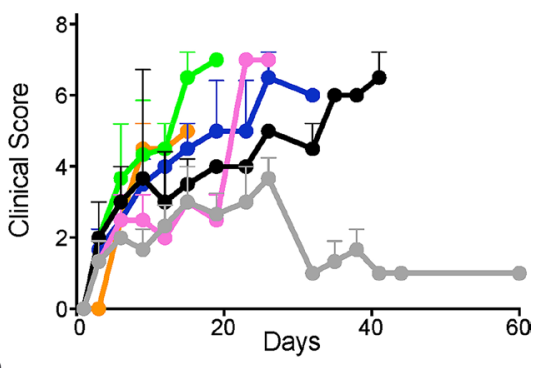

D

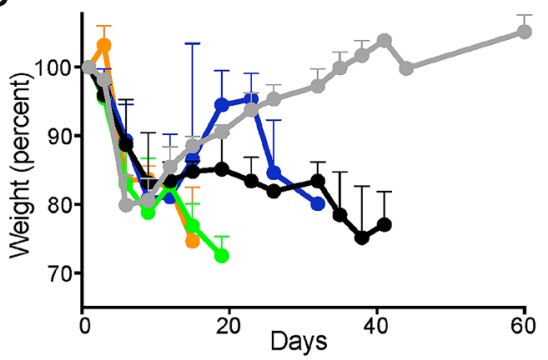

Figure 4: T cells lacking CrkL fail to eliminate a subcutaneous tumor. Lethally irradiated BALB/c mice served as recipients in this subcutaneous tumor model. As a control, irradiated mice were injected intravenously with T cell depleted C57BL/6 bone marrow alone (BM control). For experimental groups, in addition to receiving T cell depleted C57BL/6 bone marrow, irradiated mice were injected subcutaneously with A20 lymphoma cells expressing luciferase (BM+A20) with or without intravenously injected CD8 ${ }^{+} \mathrm{T}$ cells from WT, $\mathrm{DKO}, \mathrm{CrkI} / \mathrm{II} \mathrm{KO}$, or CrkL KO mice. (A) Over the next several weeks, the mice were periodically injected with luciferin and imaged to visualize tumor burden. The cross symbols represent when each individual mouse was sacrificed based on either tumor burden or severe GvHD. (B-D) Quantification of clinical data from the mice shown in panel A. (B) Overall survival ( ${ }^{*} p<0.05$, Wilcoxon test), (C) clinical score, and (D) mouse weights. One representative experiment of $n=3$ experiments, with 7 mice per group per experiment. 
$[6,29,30]$. Therefore, the ability of the $\mathrm{T}$ cell to polymerize actin, migrate, and probe the endothelium may be defining factors for the ability to cross postcapillary venules. The preferential ability of CrkL KO T cells to access lymph nodes is likely to be attributable to differences in endothelial barriers. To access lymph nodes, $\mathrm{T}$ cells must cross high endothelial venules, specialized structures that create large pockets to fully engulf transmigrating $\mathrm{T}$ cells, efficiently escorting them across the barrier [31]. This process may be much less demanding with respect to force generation by the $\mathrm{T}$ cell actin cytoskeleton.

Our studies showing that CrkL mediates integrin responses in migrating $\mathrm{T}$ cells expands upon earlier work showing that Crk proteins are important during $\mathrm{T}$ cell activation. In that setting, CrkI/II are the best studied family members, but functional redundancy has not been systematically addressed. In particular, CrkII has been documented to bind a handful of proteins downstream of the $\mathrm{T}$ cell receptor, including ZAP-70, cCbl, CasL, and even the $\mathrm{CD} 3 \zeta$ chain [32-36]. Interestingly, both CrkII and CrkL have been implicated in LFA-1 activation at the immunological synapse, but they seem to achieve this via different mechanisms. Dephosphorylation of CrkII by SHP-1 controls CrkII's ability to activate Rap1 in the integrin-rich zone of the synapse [37]. On the other hand, CrkL regulates integrin activation by pathway involving the actin nucleator WAVE2 [38]. In addition, CrkL has also been implicated in regulating WASp activation downstream of $T$ cell receptor engagement [39]. These two studies showing that $\mathrm{CrkL}$ regulates actin function at the IS, together with the work descried here showing a role for $\mathrm{CrkL}$ in actin regulation during $\mathrm{T}$ cell migration, raise the interesting possibility that $\mathrm{CrkL}$ has specialized for regulating cytoskeletal function. Indeed, there have been no reports of CrkI or CrkII regulating actin dynamics in T cells, lending support to the idea that CrkL has evolved for regulating actin dynamics in $\mathrm{T}$ cells.

Going forward, it will be interesting to identify CrkL binding partners and other signaling molecules needed for integrin-dependent actin responses and passage into inflamed tissues. Targeting these molecules could be used to more finely control $\mathrm{T}$ cell trafficking in the treatment of inflammatory diseases and for the design of nextgeneration adoptive $\mathrm{T}$ cell therapies.

\section{MATERIALS AND METHODS}

\section{Antibodies and reagents}

Anti-CD3 (2C11) and anti-CD28 (PV1) were from BioXCell. Anti-CrkL (SC-319) was from Santa Cruz. AntiCD3-FITC was from E-biosciences. Anti-Crk (\#610036), Anti-CD8-FITC, anti-CD3, anti-CD45.1, anti-H-2K ${ }^{\text {b }}$ Pacific Blue, anti-CD45.2-APC and LIVE/DEAD Fixable Aqua Dead Cell Stain Kit were from BD Bioscience. Secondary antibodies conjugated to appropriate fluorophores and AlexaFluor 488-conjugated phalloidin were obtained from Molecular Probes. Luciferin was purchased from Perkin Elmer and Gold Bio. Recombinant mouse ICAM-1-Fc was from R\&D Systems.

\section{Mice and cell culture}

Throughout this study, Crk fl/fl: CrkL fl/fl mice [19] (C57BL/6 background, $\mathrm{H}-2 \mathrm{~K}^{\mathrm{b}}$ ) were used as WT. Mice lacking all Crk proteins in $\mathrm{T}$ cells $(\mathrm{CD} 4 \mathrm{Cre}$ : $\mathrm{Crk} \mathrm{fl} / \mathrm{fl}$ : $\mathrm{CrkL}$ fl/fl mice, DKO) were described previously [7]. To generate single $\mathrm{KO}$ mice, $\mathrm{Crk}$ fl/fl: $\mathrm{CrkL}$ fl/fl mice were crossed with $\mathrm{C} 57 \mathrm{BL} / 6$ mice. Resulting single $\mathrm{Crk} \mathrm{fl} / \mathrm{fl}$ or CrkL fl: fl mice were then crossed with CD4 Cre mice, and offspring were used as a source of $\mathrm{CrkI} / \mathrm{II} \mathrm{KO}$ or CrkL $\mathrm{KO}$ T cells. BALB/c mice $\left(\mathrm{H}-2 \mathrm{~K}^{\mathrm{d}}\right)$ were used as recipients in the GvHD/GVL studies. Unless otherwise indicated, all tissue culture reagents were from Gibco. Primary mouse $\mathrm{CD}^{+} \mathrm{T}$ cells were purified from lymph nodes and spleens by negative selection using anti-MHCII and anti-CD8 hybridoma supernatants (M5/114 and 2.43, respectively) and anti-rat Ig magnetic beads (Qiagen BioMag). The resulting $\mathrm{CD}^{+} \mathrm{T}$ cells were then immediately activated on 24-well plates coated with anti-CD3 and anti-CD28 $\left(1 \mathrm{ug} / \mathrm{ml}\right.$ each) at $1 \times 10^{6}$ cells per well. Activation was done in complete $\mathrm{T}$ cell media (DMEM, 5\% FBS, penicillin/streptomycin, non-essential amino acids, Glutamax, and $55 \mu \mathrm{M} \beta$-mercaptoethanol). After $48 \mathrm{~h}$, cells were removed from activation and diluted 1:1 with complete $\mathrm{T}$ cell media containing recombinant IL-2 (25 U/ $\mathrm{mL}$ final concentration, obtained through the NIH AIDS Reagent Program, Division of AIDS, NIAID, NIH from Dr. Maurice Gately, Hoffmann - La Roche Inc [40]). T cells were used 5-6 days after activation.

\section{F-actin quantification of migrating $T$ cells}

Activated $\mathrm{CD}^{+} \mathrm{T}$ cells were resuspended in Leibovitz's L-15 media (Gibco) supplemented with $2 \mathrm{mg} / \mathrm{mL}$ glucose and incubated at $37^{\circ} \mathrm{C}$ for $20 \mathrm{~min}$. T cells were then added to ICAM- 1 coated coverslips (coated with $2 \mu \mathrm{g} / \mathrm{mL}$ overnight at $4^{\circ} \mathrm{C}$ ). After $20 \mathrm{~min}$ at $37^{\circ} \mathrm{C}$, cells were washed in L-15 and fixed in 3.7\% paraformaldehyde in PBS. Cells were then blocked and permeabilized in PSG (PBS, $0.01 \%$ saponin, $0.05 \%$ fish skin gelatin) for $20 \mathrm{~min}$, followed by $45 \mathrm{~min}$ incubation with fluorescent phalloidin (Molecular Probes) in PSG. Cells were washed in PSG, mounted, and imaged using a 63× PlanApo 1.4 NA objective on an Axiovert 200M (Zeiss) with a spinning disk confocal system (Ultraview ERS6; PerkinElmer). Four z-planes spanning a total of $0.75 \mu \mathrm{m}$ were collected at the cell-surface interface with an Orca Flash 4.0 camera (Hamamatsu). Image analysis of the rendered stacks was conducted using Velocity v6.3 software. Cells were identified using the "Find Objects" command, using a low threshold on the actin channel, and total phalloidin staining was quantified per cell based on integrated pixel intensity. 


\section{Quantification of migration on ICAM-1}

Lab-Tek 8 chamber slides (ThermoFisher) were coated with $2 \mu \mathrm{g} / \mathrm{mL}$ ICAM- 1 overnight at $4^{\circ} \mathrm{C}$. Activated $\mathrm{CD}^{+} \mathrm{T}$ cells were washed and resuspended in L-15 containing $2 \mathrm{mg} / \mathrm{mL}$ glucose. T cells were then added to the chambers, incubated 20 min, gently washed to remove all unbound cells, and imaged using a $10 \times$ phase contrast objective at $37^{\circ} \mathrm{C}$ on a Zeiss Axiovert 200M microscope equipped with an automated $\mathrm{X}-\mathrm{Y}$ stage and a Roper EMCCD camera. Time lapse images were collected every $30 \mathrm{sec}$ for $10 \mathrm{~min}$ using SlideBook 6 software (Intelligent Imaging Innovations). Movies were exported into ImageJ, and cells were tracked using the manual tracking plugin to calculate speed.

\section{Western blotting}

Proteins were separated using NuPAGE 4-12\% BisTris gels, transferred to nitrocellulose membranes $(0.45 \mu \mathrm{m}, \mathrm{BioRad})$ and blocked using LI-COR blocking buffer diluted 1:1 with PBS for $1 \mathrm{hr}$ at RT. Primary antibodies were mixed in Tris-buffered saline, $0.1 \%$ tween-20 (TBST) with 2\% BSA and membranes were probed overnight at $4^{\circ} \mathrm{C}$. After washing, membranes were incubated with fluorescent secondary antibodies and imaged using a LI-COR Odyssey system.

\section{Graft-versus-host disease (GvHD) and graft- versus-tumor (GVT) studies}

Lethally irradiated BALB/c mice ( $800 \mathrm{cGy}$ ) mice were injcted intravenously with $5 \times 10^{6} \mathrm{~T}$ cell-depleted bone marrow (TCDBM) cells with or without $2 \times 10^{6}$ FACS-sorted $\mathrm{CD}^{+} \mathrm{T}$ cells from either WT, DKO, CrkI/ II KO or CrkL KO mice. For GVT experiments, A20 B lymphoma cells transduced with luciferase were cultured as described previously [41], and $1 \times 10^{5}$ A20 cells were injected into the mice at the same time with TCDBM and $\mathrm{CD}^{+} \mathrm{T}$ cells. For skin tumor experiments, $1 \times 10^{6}$ A20 cells were injected subcutaneously. Mice were evaluated twice a week from the time of tumor injection by bioluminescence imaging using the IVIS 200 Imaging System (Xenogen) as previously described [42]. Clinical presentation of the mice was assessed 2-3 times per week using a scoring system that sums changes in 5 clinical parameters: weight loss, posture, activity, fur texture, and skin integrity [43]. Mice were euthanized when their weight dropped to $<30 \%$ of their initial body weight.

For cell sorting to obtain purified CD8+ T cells, $\mathrm{T}$ cells were purified with anti-CD8 magnetic beads using MACS columns (Miltenyi Biotec, Auburn, CA) prior to cell surface staining. FACS sorting was performed with a FACSAria cell sorter (BD Biosciences). FACS-sorted populations were typically of $>95 \%$ purity.

\section{In vivo migration assays}

Lethally irradiated BALB/c mice were injected intravenously with $5 \times 10^{6}$ WT T cell-depleted bone marrow (TCDBM). At the same time, FACS-sorted CD8 ${ }^{+}$ $\mathrm{T}$ cells from $\mathrm{H}-2 \mathrm{~K}^{\mathrm{b}+} \mathrm{CD} 45.1^{+} \mathrm{B} 6$. SJL mice were mixed at a 1:1 ratio with $\mathrm{CD} 8^{+} \mathrm{T}$ cells from with WT, DKO, $\mathrm{CrkI} / \mathrm{II} \mathrm{KO}$ or $\mathrm{CrkL} \mathrm{KO} \mathrm{H}-2 \mathrm{~K}^{\mathrm{b}+} \mathrm{CD} 45.2^{+}$mice. Seven days post transplantation, the mice were sacrificed and lymphocytes from the liver, small intestine, spleen, and skin-draining lymph nodes were isolated. Livers were perfused with PBS, dissociated, and filtered with a $70 \mu$ filter. The small intestine was washed in media, shaken in strip buffer at $37^{\circ} \mathrm{C}$ for $30 \mathrm{~min}$ to remove the epithelial cells, washed, digested with collagenase D $(100 \mathrm{mg} / \mathrm{ml})$, DNase $(1 \mathrm{mg} / \mathrm{ml})$ for $30 \mathrm{~min}$ in $37^{\circ} \mathrm{C}$, and filtered with a $70 \mu \mathrm{m}$ filter. Lymphocytes from the liver and intestines were further enriched using a $40 \%$ Percoll gradient. The cells were then analyzed for $\mathrm{CD} 45.1^{+}$and $\mathrm{CD} 45.2^{+} \mathrm{CD} 8^{+} \mathrm{T}$ cells by flow cytometry performed on an LSR-II or FACS Canto (BD Biosciences). Dead cells were excluded from analysis with LIVE/DEAD Fixable Aqua Dead Cell staining. Data were analyzed with FlowJo software (TreeStar, Ashland, OR).

\section{Statistical analysis}

Statistics were calculated using GraphPad Prism 8. Student's t-test was used to compare two groups; when more than two groups were compared, a one-way ANOVA was performed using multiple comparisons with a Tukey correction. For grouped data, a two-way ANOVA was used with a Sidak correction. For survival curves, a Wilcoxon test was used. ${ }^{*} p<0.05 ;{ }^{* *} p<0.01 ;{ }^{* * *} p<0.001$.

\section{Abbreviations}

GvHD: graft-versus-host disease; GVT: graftversus-tumor; Crk: CT10 Regulator of Kinase; ICAM-1: Intercellular Adhesion Molecule 1; VCAM-1: vascular cell adhesion molecule 1; LFA-1: Lymphocyte functionassociated antigen 1; VLA-4: very late antigen 4; SH2: Src Homology 2; SH3: Src Homology 3.

\section{Author contributions}

NHR and MM performed experiments; NHR, JKB, and MK designed experiments, analyzed the data, and wrote the manuscript.

\section{ACKNOWLEDGMENTS}

We thank members of the Burkhardt and Karimi laboratories for helpful discussions.

\section{CONFLICTS OF INTEREST}

The authors declare no conflicts of interest. 


\section{FUNDING}

This research was funded by grants from the National Blood Foundation Scholar Award to (MK) and the National Institutes of Health (NIH LRP \#L6 MD0010106 and AI130182 to MK, AI120701 to JKB). NHR is supported by NIH T32 5T32AR007442-30, as well as previous support by a Cancer Research Institute Irvington Postdoctoral Fellowship.

\section{REFERENCES}

1. Nourshargh S, Alon R. Leukocyte migration into inflamed tissues. Immunity. 2014; 41:694-707. https://doi. org/10.1016/j.immuni.2014.10.008. [PubMed]

2. Denucci CC, Mitchell JS, Shimizu Y. Integrin function in T-cell homing to lymphoid and nonlymphoid sites: getting there and staying there. Crit Rev Immunol. 2009; 29:87109. https://doi.org/10.1615/CritRevImmunol.v29.i2.10. [PubMed]

3. Kelleher D, Murphy A, Cullen D. Leukocyte functionassociated antigen 1 (LFA-1) is a signaling molecule for cytoskeletal changes in a human $\mathrm{T}$ cell line. Eur $\mathrm{J}$ Immunol. 1990; 20:2351-2354. https://doi.org/10.1002/ eji.1830201028. [PubMed]

4. Porter JC, Bracke M, Smith A, Davies D, Hogg N. Signaling through integrin LFA-1 leads to filamentous actin polymerization and remodeling, resulting in enhanced T cell adhesion. J Immunol. 2002; 168:6330-6335. https:// doi.org/10.4049/jimmunol.168.12.6330. [PubMed]

5. Smith A, Bracke M, Leitinger B, Porter JC, Hogg N. LFA1-induced $\mathrm{T}$ cell migration on ICAM-1 involves regulation of MLCK-mediated attachment and ROCK-dependent detachment. J Cell Sci. 2003; 116:3123-3133. https://doi. org $/ 10.1242 /$ jcs.00606. [PubMed]

6. Gorina R, Lyck R, Vestweber D, Engelhardt B. beta2 integrin-mediated crawling on endothelial ICAM-1 and ICAM-2 is a prerequisite for transcellular neutrophil diapedesis across the inflamed blood-brain barrier. J Immunol. 2014; 192:324-337. https://doi.org/10.4049/ jimmunol.1300858. [PubMed]

7. Roy NH, MacKay JL, Robertson TF, Hammer DA, Burkhardt JK. Crk adaptor proteins mediate actindependent $\mathrm{T}$ cell migration and mechanosensing induced by the integrin LFA-1. Sci Signal. 2018; 11. https://doi. org/10.1126/scisignal.aat3178. [PubMed]

8. Huang Y, Clarke F, Karimi M, Roy NH, Williamson EK, Okumura M, Mochizuki K, Chen EJ, Park TJ, Debes GF, Zhang Y, Curran T, Kambayashi T. CRK proteins selectively regulate $\mathrm{T}$ cell migration into inflamed tissues. J Clin Invest. 2015; 125:1019-1032. https://doi.org/10.1172/JCI77278. [PubMed]

9. Birge RB, Kalodimos C, Inagaki F, Tanaka S. Crk and CrkL adaptor proteins: networks for physiological and pathological signaling. Cell Commun Signal. 2009; 7:13. https://doi.org/10.1186/1478-811X-7-13. [PubMed]

10. Feller SM. Crk family adaptors-signalling complex formation and biological roles. Oncogene. 2001; 20:63486371. https://doi.org/10.1038/sj.onc.1204779. [PubMed]

11. Chodniewicz D, Klemke RL. Regulation of integrinmediated cellular responses through assembly of a CAS/Crk scaffold. Biochim Biophys Acta. 2004; 1692:63-76. https:// doi.org/10.1016/j.bbamcr.2004.03.006. [PubMed]

12. Li L, Guris DL, Okura M, Imamoto A. Translocation of $\mathrm{CrkL}$ to focal adhesions mediates integrin-induced migration downstream of Src family kinases. Mol Cell Biol. 2003; 23:2883-2892. https://doi.org/10.1128/ MCB.23.8.2883-2892.2003. [PubMed]

13. Antoku S, Mayer BJ. Distinct roles for Crk adaptor isoforms in actin reorganization induced by extracellular signals. J Cell Sci. 2009; 122:4228-4238. https://doi.org/10.1242/ ics.054627. [PubMed]

14. Watanabe $\mathrm{T}$, Tsuda $\mathrm{M}$, Makino $\mathrm{Y}$, Konstantinou $\mathrm{T}$, Nishihara H, Majima T, Minami A, Feller SM, Tanaka S. Crk adaptor protein-induced phosphorylation of Gab1 on tyrosine 307 via $\mathrm{Src}$ is important for organization of focal adhesions and enhanced cell migration. Cell Res. 2009; 19:638-650. https://doi.org/10.1038/cr.2009.40. [PubMed]

15. Park TJ, Curran T. Essential roles of Crk and CrkL in fibroblast structure and motility. Oncogene. 2014; 33:51215132. https://doi.org/10.1038/onc.2013.453. [PubMed]

16. Tsuda M, Tanaka S. Roles for crk in cancer metastasis and invasion. Genes Cancer. 2012; 3:334-340. https://doi. org $/ 10.1177 / 1947601912458687$. [PubMed]

17. Kumar S, Fajardo JE, Birge RB, Sriram G. Crk at the quarter century mark: perspectives in signaling and cancer. J Cell Biochem. 2014; 115:819-825. https://doi.org/10.1002/ jcb.24749. [PubMed]

18. Sriram G, Birge RB. Emerging roles for crk in human cancer. Genes Cancer. 2010; 1:1132-1139. https://doi. org/10.1177/1947601910397188. [PubMed]

19. Park TJ, Curran T. Crk and Crk-like play essential overlapping roles downstream of disabled-1 in the Reelin pathway. J Neurosci. 2008; 28:13551-13562. https://doi. org/10.1523/JNEUROSCI.4323-08.2008. [PubMed]

20. Park T, Koptyra M, Curran T. Fibroblast Growth Requires CT10 Regulator of Kinase (Crk) and Crk-like (CrkL). J Biol Chem. 2016; 291:26273-26290. https://doi.org/10.1074/jbc. M116.764613. [PubMed]

21. Guris DL, Fantes J, Tara D, Druker BJ, Imamoto A. Mice lacking the homologue of the human 22q11.2 gene CRKL phenocopy neurocristopathies of DiGeorge syndrome. Nat Genet. 2001; 27:293-298. https://doi.org/10.1038/85855. [PubMed]

22. Park TJ, Boyd K, Curran T. Cardiovascular and craniofacial defects in Crk-null mice. Mol Cell Biol. 2006; 26:62726282. https://doi.org/10.1128/MCB.00472-06. [PubMed]

23. Wysocki CA, Panoskaltsis-Mortari A, Blazar BR, Serody JS. Leukocyte migration and graft-versus-host disease. 
Blood. 2005; 105:4191-4199. https://doi.org/10.1182/ blood-2004-12-4726. [PubMed]

24. Braiman A, Isakov N. The Role of Crk Adaptor Proteins in T-Cell Adhesion and Migration. Front Immunol. 2015; 6:509. https://doi.org/10.3389/fimmu.2015.00509. [PubMed]

25. Jankowski W, Saleh T, Pai MT, Sriram G, Birge RB, Kalodimos CG. Domain organization differences explain Bcr-Abl's preference for CrkL over CrkII. Nat Chem Biol. 2012; 8:590-596. https://doi.org/10.1038/nchembio.954. [PubMed]

26. Sarkar P, Reichman C, Saleh T, Birge RB, Kalodimos CG. Proline cis-trans isomerization controls autoinhibition of a signaling protein. Mol Cell. 2007; 25:413-426. https://doi. org/10.1016/j.molcel.2007.01.004. [PubMed]

27. Sarkar P, Saleh T, Tzeng SR, Birge RB, Kalodimos CG. Structural basis for regulation of the Crk signaling protein by a proline switch. Nat Chem Biol. 2011; 7:51-57. https:// doi.org/10.1038/nchembio.494. [PubMed]

28. Nath PR, Dong G, Braiman A, Isakov N. In vivo regulation of human CrkII by cyclophilin A and FK506-binding protein. Biochem Biophys Res Commun. 2016; 470:411416. https://doi.org/10.1016/j.bbrc.2016.01.027. [PubMed]

29. Martinelli R, Zeiger AS, Whitfield M, Sciuto TE, Dvorak A, Van Vliet KJ, Greenwood J, Carman CV. Probing the biomechanical contribution of the endothelium to lymphocyte migration: diapedesis by the path of least resistance. J Cell Sci. 2014; 127:3720-3734. https://doi. org/10.1242/jcs.148619. [PubMed]

30. Carman CV, Sage PT, Sciuto TE, de la Fuente MA, Geha RS, Ochs HD, Dvorak HF, Dvorak AM, Springer TA. Transcellular diapedesis is initiated by invasive podosomes. Immunity. 2007; 26:784-797. https://doi.org/10.1016/j. immuni.2007.04.015. [PubMed]

31. Mionnet C, Sanos SL, Mondor I, Jorquera A, Laugier JP, Germain RN, Bajénoff $M$. High endothelial venules as traffic control points maintaining lymphocyte population homeostasis in lymph nodes. Blood. 2011; 118:6115-6122. https://doi.org/10.1182/blood-2011-07-367409. [PubMed]

32. Buday L, Khwaja A, Sipeki S, Farago A, Downward J. Interactions of $\mathrm{Cb}$ with two adapter proteins, Grb2 and Crk, upon T cell activation. J Biol Chem. 1996; 271:6159-6163. https://doi.org/10.1074/jbc.271.11.6159. [PubMed]

33. Sawasdikosol S, Chang JH, Pratt JC, Wolf G, Shoelson SE, Burakoff SJ. Tyrosine-phosphorylated $\mathrm{Cbl}$ binds to $\mathrm{Crk}$ after T cell activation. J Immunol. 1996; 157:110-116. [PubMed]

34. Ohashi Y, Tachibana K, Kamiguchi K, Fujita H, Morimoto C. $\mathrm{T}$ cell receptor-mediated tyrosine phosphorylation of Cas-L, a 105-kDa Crk-associated substrate-related protein, and its association of Crk and
C3G. J Biol Chem. 1998; 273:6446-6451. https://doi. org/10.1074/jbc.273.11.6446. [PubMed]

35. Gelkop $\mathrm{S}$, Isakov $\mathrm{N}$. $\mathrm{T}$ cell activation stimulates the association of enzymatically active tyrosine-phosphorylated ZAP-70 with the Crk adapter proteins. J Biol Chem. 1999; 274:21519-21527. https://doi.org/10.1074/jbc.274.31.21519. [PubMed]

36. Dong G, Kalifa R, Nath PR, Babichev Y, Gelkop S, Isakov N. Crk adaptor proteins regulate $\mathrm{CD} 3$ zeta chain phosphorylation and TCR/CD3 down-modulation in activated T cells. Cell Signal. 2017; 36:117-126. https:// doi.org/10.1016/j.cellsig.2017.04.022. [PubMed]

37. Azoulay-Alfaguter I, Strazza M, Peled M, Novak HK, Muller J, Dustin ML, Mor A. The tyrosine phosphatase SHP-1 promotes $T$ cell adhesion by activating the adaptor protein CrkII in the immunological synapse. Sci Signal. 2017; 10. https://doi.org/10.1126/scisignal.aal2880. [PubMed]

38. Nolz JC, Nacusi LP, Segovis CM, Medeiros RB, Mitchell JS, Shimizu Y, Billadeau DD. The WAVE2 complex regulates $\mathrm{T}$ cell receptor signaling to integrins via Abl- and CrkL-C3G-mediated activation of Rap1. J Cell Biol. 2008; 182:1231-1244. https://doi.org/10.1083/ jcb.200801121. [PubMed]

39. Sasahara Y, Rachid R, Byrne MJ, de la Fuente MA, Abraham RT, Ramesh N, Geha RS. Mechanism of recruitment of WASP to the immunological synapse and of its activation following TCR ligation. Mol Cell. 2002; 10:1269-1281. https://doi.org/10.1016/S10972765(02)00728-1. [PubMed]

40. Lahm HW, Stein S. Characterization of recombinant human interleukin-2 with micromethods. J Chromatogr. 1985; 326:357-361. https://doi.org/10.1016/S00219673(01)87461-6. [PubMed]

41. Edinger M, Hoffmann P, Ermann J, Drago K, Fathman CG, Strober S, Negrin RS. CD4+CD25+ regulatory T cells preserve graft-versus-tumor activity while inhibiting graftversus-host disease after bone marrow transplantation. Nat Med. 2003; 9:1144-1150. https://doi.org/10.1038/nm915. [PubMed]

42. Contag $\mathrm{CH}$, Bachmann $\mathrm{MH}$. Advances in in vivo bioluminescence imaging of gene expression. Annu Rev Biomed Eng. 2002; 4:235-260. https://doi.org/10.1146/ annurev.bioeng.4.111901.093336. [PubMed]

43. Cooke KR, Kobzik L, Martin TR, Brewer J, Delmonte J Jr, Crawford JM, Ferrara JL. An experimental model of idiopathic pneumonia syndrome after bone marrow transplantation: I. The roles of minor $\mathrm{H}$ antigens and endotoxin. Blood. 1996; 88:3230-3239. https://doi. org/10.1182/blood.V88.8.3230.bloodjournal8883230. [PubMed] 